using factor XII-depleted plasma suggest that OSCS generates these fragments by a pathway that is dependent on kallikrein activation through factor XII.

In in vivo studies, two out of six pigs that were injected with contaminated heparin samples displayed hypotension. Importantly, rapid induction of kallikrein activity was observed in all six pigs, even when no changes in blood pressure occurred.

The authors conclude that an in vitro assay for kallikrein amidolytic activity can enable easy screening for OSCS and could help to protect the global heparin supply from further contamination.

Original article Kishimoto TK et al. (2008) Contaminated heparin associated with adverse clinical events and activation of the contact system. N Engl J Med 358: 2457-2467

\section{Pregnancy within 1 year of kidney transplantation does not affect graft survival}

Kim et al. performed a retrospective study of renal transplant recipients to identify factors associated with successful pregnancy and to assess the effect of pregnancy on graft survival.

Of the 475 women of child-bearing age (15-45 years) who had undergone renal transplantation at the Asan Medical Center, Seoul, Korea, during the period 1991-2005, all 48 who became pregnant were included in the study. After the exclusion of therapeutic abortions (all except one of which were carried out for social reasons), the rate of live births was $80 \%$. Successful pregnancy was associated with a younger age at time of transplantation and at time of pregnancy, but not with immunosuppressive regimen or transplantationto-conception interval. The graft survival rate during a median post-transplantation followup period of 9.5 years (range 3.7-15.4 years), did not differ significantly from that of a control group of 187 nonpregnant female renal transplant recipients, who were matched to their pregnant counterparts with regard to age at transplantation, immunosuppressive regimen, and timing of transplantation.

The 11 women who become pregnant within 1 year of transplantation had a similar livebirth rate and 10-year graft survival rate to the 37 who conceived more than 1 year after transplantation ( $64 \%$ vs $67 \%$ and $78.8 \%$ vs $78.6 \%$, respectively). Albeit with a caveat because of the small sample size, this observation indicates that the usual advice to wait 2 years after transplantation before conceiving might be over-cautious.

Original article Kim HW et al. (2008) The experience of pregnancy after renal transplantation: pregnancies even within postoperative 1 year may be tolerable. Transplantation 85: $1412-1419$

\section{Donor CCL2 levels might adversely affect pancreas-kidney graft survival}

An Italian study has found that a high concentration of the chemokine CCL2 in the circulation of an organ donor is associated with poor outcomes of simultaneous pancreas-kidney (SPK) transplantation. CCL2 release seems to be part of an inflammatory response that occurs after brain death in the donor.

Ogliari et al. evaluated the outcome of 77 patients with type 1 diabetes and end-stage renal disease who received SPK transplants from heart-beating donors (median follow-up 76.4 months). The median serum CCL2 level in 21 healthy living kidney donors $(73 \mathrm{pg} / \mathrm{ml})$ was similar to that in 70 healthy controls (64 pg/ml), but the median level in the 77 heartbeating donors was almost five times higher $(301.8 \mathrm{pg} / \mathrm{ml})$. Multivariate analysis showed that recipients of organs from donors in the highest tertile of CCL2 levels had hazard ratios of 4.4 for pancreas loss and 4.5 for kidney loss compared with recipients from donors in the lower two tertiles. Graft loss resulted from pancreatic thrombosis and chronic kidney graft rejection. Measurements in another group of 28 SPK recipients showed that CCL2 levels in renalvein blood after revascularization correlated with donor serum CCL2 levels.

The authors suggest that donor CCL2 levels could be used to predict the risk of graft failure in SPK recipients. Although a direct causal relationship between CCL2 and graft failure has yet to be established, inhibition of CCL2 activity in donors or recipients could possibly improve the outcomes of SPK transplantation.

Original article Ogliari AC et al. (2008) High levels of donor CCL2/MCP-1 predict graft-related complications and poor graft survival after kidney-pancreas transplantation. Am J Transplant 8: 1303-1311 